\title{
Radiographic assessment of bone tunnels after anterior cruciate ligament reconstruction: A comparison of hamstring tendon and bone-patellar tendon-bone autografting technique
}

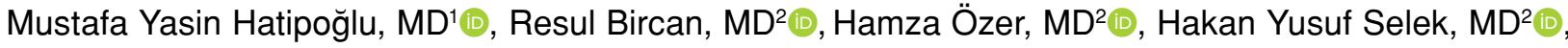 \\ Gülcan Harput, PT, $\mathrm{PhD}^{3}$ D, Yaşar Gül Baltacı, PT, $\mathrm{PhD}^{4}$ (D) \\ ${ }^{1}$ Department of Orthopedics and Traumatology, Faculty of Medicine, Pamukkale University, Denizli, Turkey \\ ${ }^{2}$ Department of Orthopedics and Traumatology, Faculty of Medicine, Gazi University, Ankara, Turkey \\ ${ }^{3}$ Department of Physiotherapy and Rehabilitation, Faculty of Health Sciences, Hacettepe University, Ankara, Turkey \\ ${ }^{4}$ Department of Physiotherapy and Rehabilitation, Ankara Güven Hospital, Ankara, Turkey
}

Anterior cruciate ligament (ACL) is the most commonly injured knee ligament and its injury can result in limitations in activities of daily living and mobility. ${ }^{[1]}$ Surgical treatment is suggested in active young athletes, in patients who have combined ligament injuries, concomitant meniscal lesions, persistent pain despite the conservative treatments, or knee instability. In this sense, anatomical reconstruction with grafting is a well-established surgical method in the treatment of ACL injury. ${ }^{[2,3]}$

Autografting, allografting, and synthetic grafting methods have been previously described for ACL

Received: April 28, 2020

Accepted: September 04, 2020

Published online: January 06, 2021

Correspondence: Mustafa Yasin Hatipoğlu, MD. Pamukkale Üniversitesi Tıp Fakültesi Ortopedi ve Travmatoloji Anabilim Dalı، 20160 Kınıklı, Denizli, Türkiye.

E-mail: mustafayasin@msn.com

Doi: $10.5606 /$ ehc. 2021.75694

Citation: Hatipoğlu MY, Bircan R, Özer H, Selek HY, Harput G, Baltacl YG. Radiographic assessment of bone tunnels after anterior cruciate ligament reconstruction: A comparison of hamstring tendon and bone-patellar tendon-bone autografting technique. Jt Dis Relat Surg 2021;32(1):122-128.

(C2021 All right reserved by the Turkish Joint Diseases Foundation

This is an open access article under the terms of the Creative Commons Attribution-NonCommercial License, which permits use, distribution and reproduction in any medium, provided the original work is properly cited and is not used for commercial purposes (http://creativecommons.org/licenses/by-nc/4.0/).

\section{ABSTRACT}

Objectives: This study aims to compare the postoperative change of femoral and tibial tunnel widths after hamstring tendon (HT) and bone-patellar tendon-bone (BPTB) autografting in primary anterior cruciate ligament (ACL) reconstruction surgery with the anteromedial portal technique.

Patients and methods: This case-control and retrospective study included 39 patients (36 males, 3 females; mean age $30.1 \pm 7.9$; range, 17 to 44 years) who underwent primary ACL reconstruction surgery with either BPTB autografting method (BPTB group, $n=18$ ) or HT autografting method (HT group, $n=21$ ) between March 2014 and December 2016. Femoral fixation was achieved with bioabsorbable screw in BPTB group and endobutton in HT group. Tibial fixation was achieved with bioabsorbable interference screw in both groups. Femoral and tibial tunnel widths of groups were compared on digital radiographs.

Results: When we compared the baseline values with the second-year results, the mean of femoral tunnel widths were significantly lower on radiographs at the second-year evaluation in both groups $(\mathrm{p}<0.001$ for all). However, the means of tibial tunnel widths were significantly lower only in the BPTB group ( $\mathrm{p}<0.001$ for BPTB group and $\mathrm{p}=0.616$ for HT group). Change levels of anteroposterior and lateral widths were more prominent in BPTB group than HT group ( $\mathrm{p}<0.001$ for all).

Conclusion: Changes in tunnel widths show us superior ossification in BPTB grafting. This can be explained by superior bone-to-bone healing. As a result of radiological evaluation, we think that BPTB grafting can be more strong and durable.

Keywords: Anterior cruciate ligament, anteromedial portal, bone-patellar tendon-bone, hamstring tendon, tunnel width.

reconstruction surgery. In the literature, no definitive conclusions have been reached about the use of synthetic grafts. ${ }^{[4,5]}$ Accordingly, autografting and allografting methods are commonly used in the 
surgical treatment of ACL. ${ }^{[6-9]}$ Although different types of autografts have been described, hamstring tendons (HTs) and bone-patellar tendon-bone (BPTB) grafting are the most commonly used techniques. ${ }^{[6-9]}$

While superior fixation and earlier healing are the advantages of BPTB grafting compared with HT autografts, anterior knee pain, donor site morbidity (patellar tendon rupture, patella fracture), or subjecting to graft-tunnel mismatch are the disadvantages. ${ }^{[8,9]}$ Less anterior knee pain, stronger biomechanical graft in the earlier periods, and being more cosmetic are the advantages of HT autografting compared with the BPTB grafting, while potential for small graft diameters (particularly in females) and increased graft laxity over time, and longer tendon-to-bone healing time are the disadvantages. ${ }^{[8,9]}$ From this point of view, we hypothesized that superior healing leads to more decreased tunnel diameter. We evaluated this with radiographic measurements. In the literature, tunnel enlargement was investigated in previous studies using various radiological techniques. ${ }^{[10-12]}$ We believe that our study will contribute to these researches by including two directions, namely, anteroposterior (AP) and lateral radiographic knee measurements. Therefore, in this study, we aimed to compare the postoperative change of femoral and tibial tunnel widths after HT and BPTB autografting in primary ACL reconstruction surgery with the anteromedial portal technique.

\section{PATIENTS AND METHODS}

This case-control and retrospective trial included 39 patients (36 males, 3 females; mean age 30.1 \pm 7.9 ; range, 17 to 44 years) who underwent primary ACL reconstruction surgery with either HT autografting method or BPTB autografting method in Faculty of Medicine, Gazi University between March 2014 and December 2016. The study protocol was approved by the Gazi University Faculty of Medicine Ethics Committee (date: 27.11.2017, number: 573). A written informed consent was obtained from each patient. The study was conducted in accordance with the principles of the Declaration of Helsinki.

The patients were allocated into two groups according to the grafting method (BPTB group [n=18] and HT group [n=21]). Femoral and tibial tunnel widths obtained from the operation notes were recorded as baseline values. In the second-year follow-up, femoral and tibial tunnel widths were measured and recorded on digital radiographs. Clinical and demographical data, and femoral and tibial tunnel widths of the groups were compared.
Patients who met any of the following criteria were excluded: secondary ACL reconstruction surgery, any other surgical method except autografting, multiple ligament injuries, concomitant cartilage lesions, and having missing data during the follow-up period. Meniscal tears, which are frequently encountered in ACL injuries, were not considered as exclusion criteria.

All surgical procedures were performed under general or spinal anesthesia by the senior surgeon. Knee joints were examined under anesthesia. Repairable meniscal injuries were sutured with all-inside sutures (FasT-Fix Meniscal Repair System, Smith \& Nephew, TN, USA). However, partial menisectomy was applied to irreparable tears such as degenerative, white-white and/or red-white zone tears.

In BPTB group, a paramedian midline incision was used for harvesting patellar tendon graft. 8 to $9 \mathrm{~cm}$ length and $1 \mathrm{~cm}$ width from the middle one-third of the patellar tendon was harvested. Bone blocks were $20 \mathrm{~mm}$ in length and about 8 to $10 \mathrm{~mm}$ in diameter on both sides.

In HT group, 2 to $3 \mathrm{~cm}$ vertical skin incision one fingerbreadth medial to tibial tuberosity was placed. Inverted L shaped incision was made over the pes tendons. Gracilis and semitendinosus tendons were harvested and the sartorius fascia was repaired. A quadrupled tendon construct was formed and the diameter of the construct was measured 7.5 to $9 \mathrm{~mm}$ (ACUFEX $^{\mathrm{TM}}$ GRAFTMASTER ${ }^{\mathrm{TM}}$ Graft Preparation System, Smith\&Nephew, Andover, Maryland).

Anteromedial portal technique was utilized for preparation of the femoral tunnel in both groups. Center of the femoral footprint was marked with an awl and the guide pin was advanced through anteromedial portal while the knee was flexed to $120^{\circ}$. Femoral tunnels were created so that the tunnel diameters were the same as the graft diameters.

In both groups, tibial tunnel was created using the remnants of the tibial footprint as a guide via standard approach $1 \mathrm{~mm}$ greater than the measured diameter of the graft. Tibial tunnel was created over the guide pin, which was placed at a $47.5^{\circ}$ angle to the tibial plateau in sagittal plane.

In BPTB group, the femoral tunnel was created at a depth that could enter a $20 \mathrm{~mm}$ graft. The patellar tendon graft with bone block was pulled from the tibial tunnel into the femoral tunnel. A bioabsorbable interference screw, which had a $1 \mathrm{~mm}$ smaller diameter than the femoral tunnel diameter, was placed for graft 
fixation. In HT group, the femoral tunnel was created at a depth that could enter a $25 \mathrm{~mm}$ graft. Femoral fixation of the hamstring tendons was achieved with endobutton (EndoButton ${ }^{\circledR}$ CL Ultra, Smith \& Nephew, Memphis, Tennessee, USA).

Fixation on the tibial side was achieved with bioabsorbable interference screw of which the diameter was the same as the tibial tunnel diameter. No supplemental fixation was performed.

First-generation cephalosporin $1 \mathrm{~g}$ was administered intravenously to all patients every $8 \mathrm{~h}$ in the first $24 \mathrm{~h}$ postoperatively. Daily $4000 \mathrm{IU} / \mathrm{mL}$ enoxaparin was administered subcutaneously for 10 days for prophylaxis of deep venous thrombosis. Orthosis was not given and early rehabilitation was encouraged with crutches; however, full weight bearing was initiated as the patient tolerated. The same treatment and rehabilitation protocol was applied to both groups.

Anteroposterior and lateral knee radiographs were used to assess the width of femoral and tibial tunnels. Lateral radiographs were administered while the knee was in $30^{\circ}$ of flexion. The magnification factor was not used since the visual size would be 1:1 because it was a standardized digital radiography (Ralco R225 Collimator, Ralco X-Ray Equipment, Biassono, Italy). Digital radiographs of the patients were evaluated on a computer screen using the Enlil PACS System-2.5 (Enlil PACS Viewer, Eroglu Yazılım, Eskisehir, Turkey). Radiographic measurements were performed on second-year follow-up radiographs. To ensure the inter-observer reliability, measurements were performed twice by two different orthopedic surgeons at intervals of two weeks and the values obtained were averaged. Thus, possible technical errors were minimized. Sclerotic margins nearby the tunnels were accepted as the landmarks. Diameter measurements were performed at the beginning of the tunnel, end of the tunnel, and middle of the tunnel (Figure 1). First, measurements were performed from the midpoint of the tunnel and then from the starting point of the tunnel and the endpoint of the tunnel at an equal distance to the midpoint. The mean value of three consecutive measurements was considered for analyses. This measurement technique was implemented in previous studies. ${ }^{[12-15]}$ When comparing BPTB and HT groups, the mean widths of the femoral and tibial tunnels were used.

\section{Statistical analysis}

Statistical analysis was performed using the IBM SPSS version 21.0 software (IBM Corp., Armonk, NY, USA). Descriptive data were given as mean, standard deviation, median, quartiles, count (n) or percentage $(\%)$. Categorical variables were compared with chi-square or Fischer's exact test. After checking the normal distribution, between-group comparisons were performed using Mann-Whitney $U$ test or Student's t-test. Baseline and after-surgery data within the group were used using paired t-test or Wilcoxon signed-rank test. The confidence value of 95\% was selected and $p$ value of 0.05 was accepted as statistically significant.

\section{RESULTS}

Clinical and demographical features of the groups are shown in Table I. No significant difference was observed between the groups in terms of age, sex, and involved side ( $p>0.05$ for all). In the BPTB group, two male patients had bleeding at the wound site in the early postoperative period, without further complications. A total of 10 patients underwent meniscus repair in both groups, and four patients underwent partial menisectomy. No graft rupture was observed during follow-up period.

For all measurements, intra-observer reliability ranged between 0.924 and 0.978, while interobserver reliability ranged between 0.817 and 0.910 . Measurements had good inter- and intra-observer consistency.

Baseline values of femoral and tibial tunnel widths were significantly higher in the BPTB group compared with the HT group ( $\mathrm{p}<0.05$ for both).

In second-year follow-up, mean of femoral tunnel widths on AP view changed from 8.7 to $4 \mathrm{~mm}$ and from 8.0 to $6.7 \mathrm{~mm}$ in BPTB and HT groups,
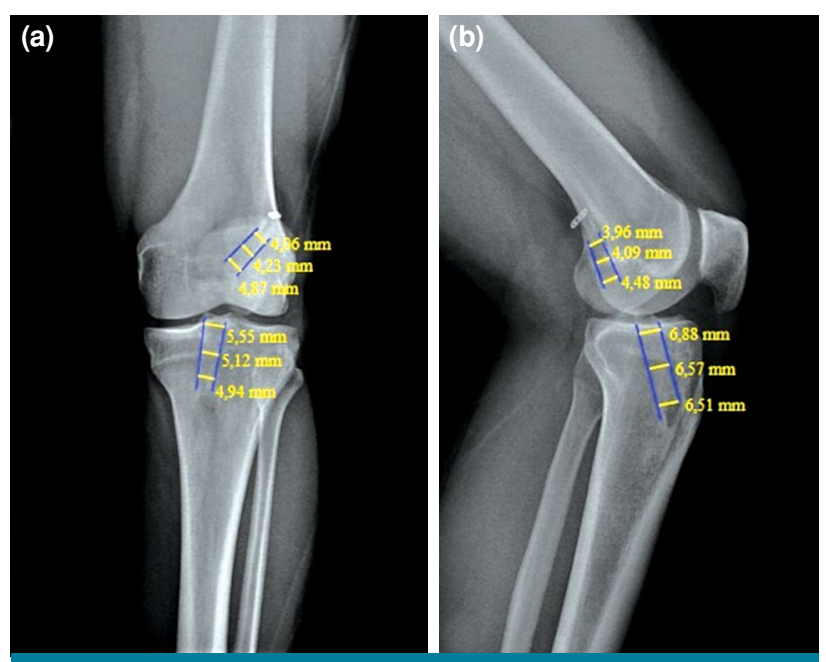

FIGURE 1. (a) Anteroposterior and (b) lateral radiographs show femoral and tibial tunnel diameter measurements. 


\begin{tabular}{|c|c|c|c|c|c|c|c|c|c|c|c|}
\hline \multirow[b]{3}{*}{ Variables } & \multicolumn{10}{|c|}{$\begin{array}{c}\text { TABLE I } \\
\text { Clinical and demographical features }\end{array}$} & \multirow[b]{3}{*}{$p$} \\
\hline & \multicolumn{5}{|c|}{ BPTB group $(n=18)$} & \multicolumn{5}{|c|}{ HT group $(n=21)$} & \\
\hline & $\mathrm{n}$ & $\%$ & Mean $\pm S D$ & Median & $\begin{array}{l}25^{\text {th }}-75^{\text {th }} \\
\text { Percentile }\end{array}$ & $\mathrm{n}$ & $\%$ & Mean \pm SD & Median & $\begin{array}{l}25^{\text {th }}-75^{\text {th }} \\
\text { Percentile }\end{array}$ & \\
\hline Age (year) & & & $29.5 \pm 7.9$ & & & & & $30.5 \pm 8.0$ & & & 0.679 \\
\hline Sex & & & & & & & & & & & 0.052 \\
\hline Male & 15 & 83.3 & & & & 21 & 100 & & & & \\
\hline Female & 3 & 16.7 & & & & 0 & 0 & & & & \\
\hline Involved side & & & & & & & & & & & 0.757 \\
\hline Right & 12 & 66.7 & & & & 13 & 61.9 & & & & \\
\hline Left & 6 & 33.3 & & & & 8 & 38.1 & & & & \\
\hline Femoral tunnel (mm) & & & & & & & & & & & \\
\hline Baseline AP/lateral mean width & & & $8.7 \pm 0.6$ & & & & & $8.0 \pm 0.4$ & & & $<0.001$ \\
\hline $2^{\text {nd }}$ year AP mean width & & & $4.0 \pm 2.2$ & & & & & $6.7 \pm 1.2$ & & & $<0.001$ \\
\hline $2^{\text {nd }}$ year lateral mean width & & & $3.6 \pm 1.9$ & & & & & $6.2 \pm 1.2$ & & & $<0.001$ \\
\hline Diameter change value (AP) & & & & 3.94 & $3.09-6.18$ & & & & 1.19 & $0.26-2.21$ & $<0.001$ \\
\hline Diameter change value (lateral) & & & $5.14 \pm 2.3$ & & & & & $1.76 \pm 1.1$ & & & $<0.001$ \\
\hline Tibial tunnel (mm) & & & & & & & & & & & \\
\hline Baseline AP/lateral mean width & & & $9.4 \pm 0.6$ & & & & & $8.6 \pm 0.5$ & & & $<0.001$ \\
\hline $2^{\text {nd }}$ year AP mean width & & & $7.1 \pm 2.7$ & & & & & $8.7 \pm 0.9$ & & & 0.029 \\
\hline $2^{\text {nd }}$ year lateral mean width & & & $6.6 \pm 2.6$ & & & & & $8.7 \pm 0.9$ & & & 0.006 \\
\hline Diameter change value (AP) & & & & 1.83 & $0.33-3.06$ & & & & -0.16 & $-0.66-0.56$ & $<0.001$ \\
\hline Diameter change value (lateral & & & & 2.09 & $0.38-3.72$ & & & & 0 & $-0.74-0.62$ & $<0.001$ \\
\hline
\end{tabular}

respectively. Mean of femoral tunnel widths on lateral view changed from 8.7 to $3.6 \mathrm{~mm}$ and from 8.0 to $6.2 \mathrm{~mm}$ in BPTB and HT groups, respectively.

In second-year follow-up, mean of tibial tunnel widths on AP view changed from 9.4 to $7.1 \mathrm{~mm}$ and from 8.6 to $8.7 \mathrm{~mm}$ in BPTB and HT groups, respectively. Mean of tibial tunnel widths on lateral view changed from 9.4 to $6.6 \mathrm{~mm}$ and from 8.6 to 8.7 $\mathrm{mm}$ in BPTB and HT groups, respectively (Figure 2).
When we compared the baseline values with the second-year results, the mean of femoral tunnel widths was significantly lower on radiographs at the second-year evaluation in both groups $(p<0.001$ for all). However, the mean of tibial tunnel widths was significantly lower only in the BPTB group $(p<0.001$ for BPTB group and $p=0.616$ for HT group). In BPTB group, change levels of $\mathrm{AP}$ and lateral widths were more prominent than HT group $(\mathrm{p}<0.001$ for all).
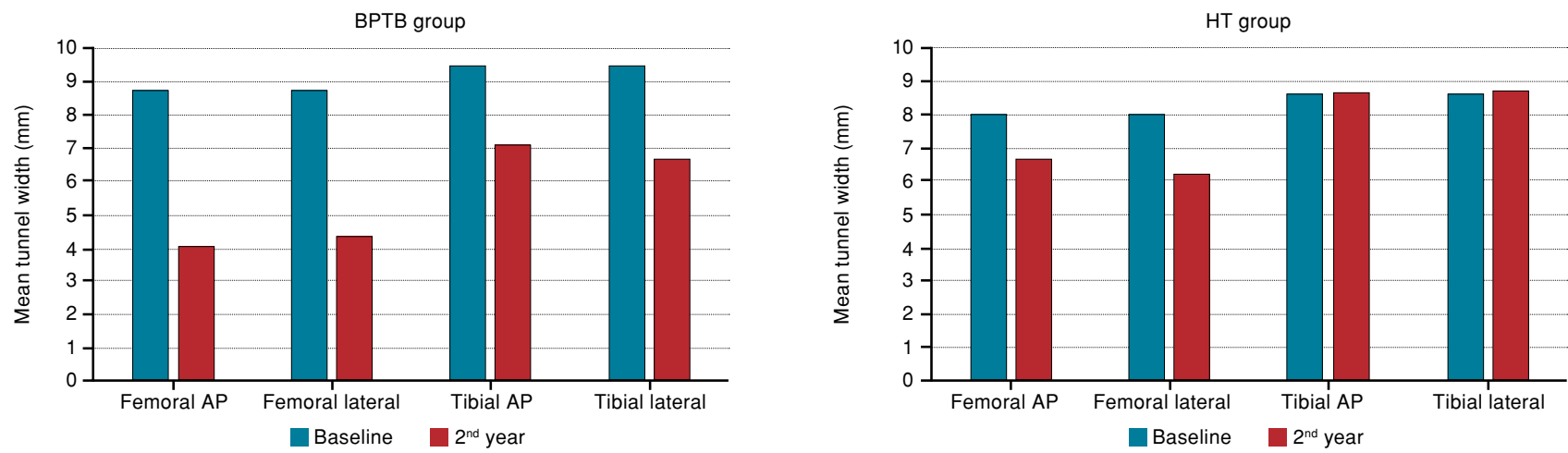

FIGURE 2. Graphs show baseline (mean) and second-year follow-up (mean) measurements (in mm) of femoral and tibial tunnels. BPTB: Bone-patellar tendon-bone; HT: Hamstring tendon; AP: Anteroposterior. 


\section{DISCUSSION}

In this study, we aimed to compare the postoperative changes in femoral and tibial tunnel widths after HT grafting and BPTB grafting in primary ACL reconstruction surgery. The most significant result of this study is that femoral tunnel AP and lateral view widths were significantly lower at the second-year evaluation in both groups. The change levels were more prominent in the BPTB group than the HT group. Secondly, tibial tunnel AP and lateral view widths reduced only in the $\mathrm{BPTB}$ group, indicating that the BPTB grafting heals superiorly radiologically.

The purpose of ACL reconstruction is to place a graft in accordance with the ACL physiology and anatomy by opening a tunnel in the tibia and femur. After the graft has been placed, it undergoes some physiological and biomechanical changes and resembles the original ACL. ${ }^{[16,17]}$ Once the graft is placed in the tunnel, it passes through stages such as synovialization, neovascularization, and ligamentization, i.e. remodeling process, and then integrates into the bone structure. ${ }^{[16,17]}$ Since the grafts are normally avascular structures, tendon healing in a bone tunnel requires bone ingrowth into the tendon. Sharpey's fiber formation and fibrocartilage fixation provide the healing process. ${ }^{[18]}$ BPTB grafts show superior fixation compared to HT grafts because of the bone-to-bone healing. ${ }^{[8,9]}$ From this point of view, we thought that the tunnel widths would be lower in BPTB grafting than HT grafting. Besides, we believe that a follow-up period of two years is acceptable for the fixation of the grafts.

Different imaging methods such as digital radiographs, two-dimensional computed tomography (CT), three-dimensional (3D) CT or magnetic resonance imaging (MRI) can provide data about femoral and tibial tunnels after ACL surgery. ${ }^{[19,20]}$ Exposure to higher doses of radiation (for $\mathrm{CT}$ ), requiring longer examining time, and higher costs are the main disadvantages of CT and MRI. In addition, digital radiographs have been previously shown to be a valid and reliable imaging method to assess the femoral and tibial tunnel size. ${ }^{[12]}$ Therefore, in our study, we have used digital radiographs.

There are some studies evaluating the tunnel size after ACL reconstruction in the pertinent literature. Tunnel enlargement is a common finding in the postoperative period and although it is not clearly known, osteolysis due to the inflammatory process is an important reason for this. ${ }^{[21,22]}$ Laxdal et al. ${ }^{[15]}$ randomized 77 patients who underwent
HT autografting surgery into two groups. While metallic interference screw was used for fixation in the first group, biodegradable interference screw was used in the second group. According to their results, there were significantly larger radiographically visible drill holes on both the tibial and femoral sides in the biodegradable group compared with the metal group at the sixth and $24^{\text {th }}$ months. Moreover, Karikis et al. ${ }^{[12]}$ assessed the tibial tunnel at secondand fifth-year radiographs after ACL reconstruction using HT autografts and biocomposite interference screw. In $83 \%$ of patients, the width of the tibial tunnel had decreased on the radiographic views. Furthermore, Frosch et al. ${ }^{[11]}$ performed MRI imaging on 12 patients at 3, 6, and 12 months after surgery, who underwent ACL surgery using biocomposite screws. They reported that the biocomposite screw dissolved in the first six months and the tunnel width decreased after 12 months. According to our results, the baseline values of femoral and tibial tunnel widths were higher in the BPTB group because the bone grafts generally require a wider tunnel. After the surgery, the mean of femoral tunnel widths on radiographs decreased in both groups. However, while the tibial tunnel widths decreased in the ВРТВ group, they increased in the HT group. Change levels of AP and lateral widths were more prominent in BPTB group. ${ }^{[23]}$ Graft selection is an important factor in tunnel enlargement, and it has been previously shown that tendons cause more tunnel expansion than bone blocks. ${ }^{[14,23]}$ Park et al. ${ }^{[24]}$ reported that bone-to-bone healing is better in the rabbit model. We believe that this fact could be attributed to the superior healing process of BPTB grafting. Previous studies evaluating tunnel width according to graft selection in ACL reconstruction have similarly reported that tunnel enlargement is more common with HT grafting. ${ }^{[10,23,25]}$

In the BPTB group, the tibial tunnel narrowed less than the femoral tunnel, while in the HT group, the tibial tunnel diameter was measured higher than the baseline values. Bone mineral density may cause this situation. In some studies evaluating bone mineral density after knee surgery (ACL, meniscus surgery), it was reported that bone density in the distal femur was higher than the proximal tibia in the postoperative period. ${ }^{[26,27]}$ We believe that there is more narrowing in femoral tunnels because higher bone density is more resistant to tensile forces.

This study has some limitations. Firstly, the study had a retrospective design and a limited sample size. Second and the most important deficiency was not evaluating functional parameters and activity scores. Also, a two-year follow-up can only show us early 
period results. Moreover, although radiographs are easily accessible and cost-effective enabling tunnel width measurement to be practical and easy, in reality, they do not reflect the 3D structure and volume of the tunnels. Therefore, some studies have shown that they can reveal measurements lower than the actual tunnel diameter. ${ }^{[23]}$ It is also difficult to identify tunnels on radiography in long-term follow-up. ${ }^{[28]}$

In conclusion, this study has shown radiologically that the BPTB grafting heals superiorly compared to HT grafting in primary ACL reconstruction surgery with the anteromedial portal technique. This may gain more strenght and durability to the BPTB grafting. Therefore, BPTB grafting may be a better option for young and active athletes. Further studies evaluating the tunnel sizes in cohort designs considering the functional parameters are awaited.

\section{Declaration of conflicting interests}

The authors declared no conflicts of interest with respect to the authorship and/or publication of this article.

\section{Funding}

The authors received no financial support for the research and/or authorship of this article.

\section{REFERENCES}

1. Atik OŞ. Surgical versus conservative treatment for torn anterior cruciate ligament. Jt Dis Relat Surg 2020;31:159-60.

2. Vyas D, Rabuck SJ, Harner CD. Allograft anterior cruciate ligament reconstruction: indications, techniques, and outcomes. J Orthop Sports Phys Ther 2012;42:196-207.

3. Muller B, Hofbauer M, Wongcharoenwatana J, Fu FH. Indications and contraindications for double-bundle ACL reconstruction. Int Orthop 2013;37:239-46.

4. Longo UG, Rizzello G, Berton A, Fumo C, Maltese L, Khan WS, et al. Synthetic grafts for anterior cruciate ligament reconstruction. Curr Stem Cell Res Ther 2013;8:429-37.

5. Legnani C, Ventura A, Terzaghi C, Borgo E, Albisetti W. Anterior cruciate ligament reconstruction with synthetic grafts. A review of literature. Int Orthop 2010;34:465-71.

6. Dhammi IK, Rehan-Ul-Haq, Kumar S. Graft choices for anterior cruciate ligament reconstruction. Indian J Orthop 2015;49:127-8.

7. Genuario JW, Faucett SC, Boublik M, Schlegel TF. A cost-effectiveness analysis comparing 3 anterior cruciate ligament graft types: bone-patellar tendon-bone autograft, hamstring autograft, and allograft. Am J Sports Med 2012;40:307-14.

8. Koh E, Oe K, Takemura S, Iida H. Anterior cruciate ligament reconstruction using a bone-patellar tendon-bone autograft to avoid harvest-site morbidity in knee arthroscopy. Arthrosc Tech 2015;4:e179-84.

9. Frank RM, Higgins J, Bernardoni E, Cvetanovich G, BushJoseph CA, Verma NN, et al. Anterior cruciate ligament reconstruction basics: Bone-patellar tendon-bone autograft harvest. Arthrosc Tech 2017;6:e1189-e94.

10. Amano H, Tanaka Y, Kita K, Uchida R, Tachibana Y, Yonetani $\mathrm{Y}$, et al. Significant anterior enlargement of femoral tunnel aperture after hamstring ACL reconstruction, compared to bone-patellar tendon-bone graft. Knee Surg Sports Traumatol Arthrosc 2019;27:461-70.

11. Frosch KH, Sawallich T, Schütze G, Losch A, Walde T, Balcarek $\mathrm{P}$, et al. Magnetic resonance imaging analysis of the bioabsorbable Milagro interference screw for graft fixation in anterior cruciate ligament reconstruction. Strategies Trauma Limb Reconstr 2009;4:73-9.

12. Karikis I, Ejerhed L, Sernert N, Rostgård-Christensen L, Kartus J. Radiographic tibial tunnel assessment after anterior cruciate ligament reconstruction using hamstring tendon autografts and biocomposite screws: a prospective study with 5-year follow-up. Arthroscopy 2017;33:2184-94.

13. Peyrache MD, Djian P, Christel P, Witvoet J. Tibial tunnel enlargement after anterior cruciate ligament reconstruction by autogenous bone-patellar tendon-bone graft. Knee Surg Sports Traumatol Arthrosc 1996;4:2-8.

14. L'Insalata JC, Klatt B, Fu FH, Harner CD. Tunnel expansion following anterior cruciate ligament reconstruction: a comparison of hamstring and patellar tendon autografts. Knee Surg Sports Traumatol Arthrosc 1997;5:234-8.

15. Laxdal G, Kartus J, Eriksson BI, Faxén E, Sernert N, Karlsson J. Biodegradable and metallic interference screws in anterior cruciate ligament reconstruction surgery using hamstring tendon grafts: prospective randomized study of radiographic results and clinical outcome. Am J Sports Med 2006;34:1574-80.

16. Youn I, Jones DG, Andrews PJ, Cook MP, Suh JK. Periosteal augmentation of a tendon graft improves tendon healing in the bone tunnel. Clin Orthop Relat Res 2004;419:223-31.

17. Jackson DW, Corsetti J, Simon TM. Biologic incorporation of allograft anterior cruciate ligament replacements. Clin Orthop Relat Res 1996;324:126-33.

18. Chen $\mathrm{CH}$. Graft healing in anterior cruciate ligament reconstruction. Sports Med Arthrosc Rehabil Ther Technol 2009;1:21.

19. Illingworth KD, Hensler D, Working ZM, Macalena JA, Tashman S, Fu FH. A simple evaluation of anterior cruciate ligament femoral tunnel position: the inclination angle and femoral tunnel angle. Am J Sports Med 2011;39:2611-8.

20. Yoon SJ, Yoon YC, Bae SY, Wang JH. Bone Tunnel Diameter Measured with CT after Anterior Cruciate Ligament Reconstruction Using Double-Bundle Auto-Hamstring Tendons: Clinical Implications. Korean J Radiol 2015;16:1313-8.

21. Stolarz M, Ficek K, Binkowski M, Wróbel Z. Bone tunnel enlargement following hamstring anterior cruciate ligament reconstruction: a comprehensive review. Phys Sportsmed 2017;45:31-40.

22. Stener S, Ejerhed L, Sernert N, Laxdal G, RostgårdChristensen L, Kartus J. A long-term, prospective, randomized study comparing biodegradable and metal interference screws in anterior cruciate ligament reconstruction surgery: radiographic results and clinical outcome. Am J Sports Med 2010;38:1598-605.

23. Atik OS. Is there something new and interesting in my article?. Eklem Hastalik Cerrahisi. 2019;30:69.

24. Webster KE, Chiu JJ, Feller JA. Impact of measurement error in the analysis of bone tunnel enlargement after anterior cruciate ligament reconstruction. Am J Sports Med 2005;33:1680-7.

25. Park MJ, Lee MC, Seong SC. A comparative study of the healing of tendon autograft and tendon-bone autograft using patellar tendon in rabbits. Int Orthop 2001;25:35-9. 
26. Clatworthy MG, Annear P, Bulow JU, Bartlett RJ. Tunnel widening in anterior cruciate ligament reconstruction: a prospective evaluation of hamstring and patella tendon grafts. Knee Surg Sports Traumatol Arthrosc 1999;7:138-45.

27. van Meer BL, Waarsing JH, van Eijsden WA, Meuffels DE, van Arkel ER, Verhaar JA, et al. Bone mineral density changes in the knee following anterior cruciate ligament rupture. Osteoarthritis Cartilage 2014;22:154-61.
28. van der Wal RJP, Attia D, Waarsing EH, Thomassen BJW, van Arkel ERA. Two-year follow-up of bone mineral density changes in the knee after meniscal allograft transplantation: Results of an explorative study. Knee 2018;25:1091-9.

29. Fink C, Zapp M, Benedetto KP, Hackl W, Hoser C, Rieger $\mathrm{M}$. Tibial tunnel enlargement following anterior cruciate ligament reconstruction with patellar tendon autograft. Arthroscopy 2001;17:138-43. 